\title{
Stunting in India: An Empirical Approach to Human Rights-Based Solutions
}

\author{
Rupal Parekh $^{1}$ - Vijayan K. Pillai ${ }^{1}$
}

Published online: 8 December 2016

(C) Springer International Publishing 2016

\begin{abstract}
This study was undertaken to understand the relationship between poor sanitation and hygiene, clean drinking water, toilet use, mother's education, and religious practices and stunting. A human rights theoretical framework was utilized for this study. The purpose of this paper is to examine the causal linkages between childhood stunting and determinants such as religion and mother's education. The study used data from the Third National Family Health Survey (NFHS-3; International Institute for Population Sciences, 2006). The sample consisted of 17,239 women between the ages of 15 and 49 with a child between the ages of 2 and 5. The dependent variable, stunting, is dichotomized to create stunted and non-stunted groups. Logistic regression and factorial ANOVA were used to test how access to clean drinking water, toilet use, mother's education, and religious practices impact childhood stunting. International social work efforts should focus on working with social institutions that improve women's status, and enlisting their participation in public sanitation campaigns will potentially save India from losing its most valuable resource - children. This study pioneers an empirical approach toward developing interventions based on a human rights approach. The approach illustrated in this study may be replicated for developing social work interventions for any number of social problems, which may be cast as a human rights issue.
\end{abstract}

Vijayan K. Pillai

Pillai@uta.edu

Rupal Parekh

Rupal.Parekh@mavs.uta.edu

1 School of Social Work, University of Texas-Arlington, 211 South Cooper Street, Arlington, TX 76019, USA
Keywords Stunting $\cdot$ Human rights $\cdot$ Sanitation $\cdot$ Religion $\cdot$ Ecological

The associations among key characteristics of poor cognitive, motor, and social development among children have been a subject of concern to the development agenda among countries of the South (Victora et al. 2008). Studies have shown that there is a direct link to childhood stunting and lower test scores, the loss of economic productivity, reduction of schooling, and increased risk of degenerative disease such as diabetes (Stillwaggon 1998). The World Health Organization has recently recognized that childhood stunting is not caused by dietary insufficiencies and inadequacies alone, and economic, social, and environmental practices and structures play a critical role in the increased number of stunted children throughout the world (Singer and Erickson 2013).

India has the largest number of stunted children (Kanjilal et al. 2010). Although studies have shown that socioeconomic factors play a critical role in the percentage of stunted children in India, it is important to note that $48 \%$ of children in India are stunted across economic groups (Gulati 2010). Given the overall economic growth throughout India, it is shocking that percentages of stunted children across all income groups are comparable to similar percentages in sub-Saharan Africa where the GDP is much worse (Panagariya 2013). This suggests that stunting is a systemic problem that requires immediate attention by policy makers and influential decision-makers.

A loss of human potential of the magnitude of $50 \%$ has tremendous socioeconomic consequences on a society that struggles to meet the basic needs of its citizens. Against this backdrop of widespread pessimistic assessment of stunting in India, small-scale improvements in localized regional pockets are slowly beginning to emerge. Maharashtra, a western state in India, successfully reduced stunting rates in children 
younger than 2 years, from $44 \%$ in 2005 to $22.8 \%$ in 2012 (Haddad et al. 2014). The secret of their success lies in the development of a technical advisory body, which provides the government with evidence-based interventions. These interventions have played a critical role in educating a vast majority of the public who had deep-seated beliefs about appropriate sanitation practices that often were informed by cultural and religious practices. Improvement in living and health conditions and a substantial decline in childhood mortality are a few of the factors that have positively impacted children's status in a country that previously struggled to provide children their basic rights. The need to increase attention and awareness so that action and cost-effective interventions and polices are implemented is critical due to the increasing numbers of children at risk of negative life course outcomes (Pillai and Parekh 2015).

Growing evidence suggests a link between poor sanitation and hygiene, clean drinking water, toilet use and access, mother's education, and religious practices. These critical factors play a vital role in understanding an epidemic that is currently robbing nations, like India, of their human potential (Aguayo et al. 2016).

Sanitary practices play a critical role in building healthy communities. According to a recent article in the New York Times, in India, the issue is not a lack of food, like previously believed, rather a lack of toilet facilities for its 620 million people who defecate outside (Spears et al. 2013). Furthermore, children who do not have clean drinking water may ingest bacteria causing intestinal infection, which has been linked to stunting and anemia and puts children at risk of developmental disabilities (Spears et al. 2013).

Since the 1920s, leaders like Gandhi have spoken about the dire need to improve sanitary conditions in India. Gandhi famously said, "Sanitation is more important than independence." However, his efforts fell on deaf ears, as currently over 620 million Indians continue to defecate outside. Although there are current political campaigns and a growing body of research addressing this issue, cultural and religious practices and beliefs serve as protective factors against childhood stunting in certain Indian states while they contribute to the growing number of children with infectious diseases that often lead to their death in other states. However, a recent study has pointed to generational difference rather than idiosyncratic differences between Hindus and Muslims as the leading contributor to the differences in sanitary practices and childhood stunting (Geruso and Spears 2014).

Studies utilizing a sociobiological perspective in particular have vastly contributed toward explaining the linkages among sanitation, diarrheal diseases, and stunting. The focus on the sociobiological pathways to stunting has to an extent resulted in the neglect of the religio-cultural determinants of stunting in India (Brainerd and Menon 2015). In this regard, this study has two objectives. The first is to examine the effect of religion on stunting net of demographic and environmental factors often associated with stunting in India. The second objective is conditional on the findings related to the role of religion and other selected determinants on stunting. If religion/cultural influences are significant, our second objective is to cast our findings on stunting within a human rights perspective with implications for designing social interventions for reducing the likelihood of stunting in India.

\section{Modeling Stunting in India}

Recently, a study explored over 100 rural districts in India and found a strong association between the prevalence of open defecation and stunting (Spears et al. 2013). Although this analysis added to the growing body of research, further work in this area about the behavioral and cultural practices that influence and sustain such behaviors as open defecation will strengthen the evidence needed to influence the development of policy which inform programmatic intervention, activities, and monitoring.

Behavioral changes are hard to accomplish, especially when they are directly related to the religious beliefs of a community. The attitudes toward purity and pollution, cleanliness, and hygiene are considered sacred in nature in most religious communities in India. Some of these age-old practices have perhaps unintentionally impacted the children's health status negatively in Hindu communities. Banda et al. (2007) found that Indian residents in a rural village in Harijan colony (a Hindu community) did not associate unsafe stored drinking water with the development of diarrheal disease, rather attributing it to "heat," spicy food, ingesting hair, mud, or mosquitoes.

Muslim populations across India have lower childhood mortality, although Muslims on average are poorer and less educated than Hindus (Bhalotra et al. 2010). This puzzling phenomenon is largely due to the differences in cultural practices that govern cleanliness in Muslim and Hindu communities. Since India is highly segregated, most Muslim communities live in their own areas, which further protects them from bacteria and worms contained in feces that get transmitted via contact with the skin (Bhalotra et al. 2010). Since low-caste individuals from Hindu communities often have been tasked to clean human waste, there are a large percentage of untouchable children with diarrheal disease (Geruso and Spears 2014).

The differences in the effects of religion on stunting are often attributed to the dissimilarities in values and norms that guide lifestyles and contribute to beliefs with regard to health and well-being. Within India, Hinduism is seldom regarded as a monolithic organized system of religious values and beliefs. There are vast differences in the practice of the religion across language groups and geographic regions. Much of this 
variation within Hinduism may be attributed to a system of social differentiation, the caste system. More recently, several social scientists (Thorat and Newman 2007; Chaudhury 2004) have argued that caste differences indicative of access to traditional power within the religion should be taken into consideration in assessing the role of religion on outcomes such as stunting. Deshpande (2001) offers a caste disparity index based on five social and economic characteristics, occupation, education, land holding, assets, and livestock. The caste disparity index measures the level of social distance among various caste groups within Indian states. Variables, which measure the influence of caste hierarchies on stunting, are to be controlled to assess the net effect of religion on stunting (Som et al. 2007).

Educational interventions play a crucial role in shedding light on issues that are greatly ignored due to fear in a largely castedriven society that supports the existence of a voiceless, marginalized subpopulation. These individuals have limited modern resources and have little to no access to knowledge and power that could potentially influence their social, cultural, and political awareness and practices. Educational interventions within a human rights framework call for a sound understanding of the cultural factors related to stunting in order to limit the prevalence of stunting in India (Bose et al. 2007).

Building on the existing literature in our first hypothesis, we believe that cultural, structural, economic, and sanitary practices will play a significant role in the stunting of children between the ages of 2 and 5. More specifically, we argue that the likelihood of a child between the ages of 2 and 5 years being stunted is significantly associated with factors such as clean water (piped) and use of toilet (flush or pit). We identify two groups of factors, one demographic and the other environmental. The demographic group is composed of two determinants, religion and the mother's level of education. The environmental group has two factors, access to clean water and availability of flush or pit toilet.

The two hypotheses related to the demographic determinants are as follows. We predict that religion (Hindu, non-Hindu) and women's education will influence children's level of stunting. More specifically, Hindu families will have higher rates of stunting among children than non-Hindu families. Although $48 \%$ of children across income groups are stunted in India, caste continues to be a great divide among Hindu populations. Stunting rates among Hindu children, the so-called low-caste untouchables, is disproportionately high, due to being tasked with cleaning human waste. Since non-Hindu populations do not have caste systems, which often govern occupational activities, lowincome individuals from other religious groups are not commissioned to do such work (Maruthi and Busenna 2015). In India, the level of a mother's education is often directly tied to her socioeconomic status. Mothers that have completed a secondary education will be less likely to have stunted children than mothers that have not completed a secondary education.

The hypotheses associated with the environmental set are as follows. First, families that have access to a toilet (flush or pit) are less likely to have stunted children than those without a flush or pit toilet. Furthermore, it is hypothesized that families with clean drinking water (piped and tube) are less likely to have stunted children than those without clean drinking water. Prior studies point to a significant association between toilet type and educational status (Shah and Bali 2015). Mothers with secondary education are more likely to be aware of the hazards of defecation in the open and are more likely to muster resources either directly or indirectly to secure flush- or pit-type toilets which will provide her high levels of sanitation and privacy (Pillai and Parekh 2015). .

\section{Method}

\section{Data Source}

Data from the Third National Family Health Survey (NFHS3) of India are used in this study (International Institute for Population Sciences 2007). The NFHS-3 is a large-scale, multi-round sample survey of households throughout India, which includes a nationally representative sample of 124,385 women of ages 15-49 and 74,369 men of ages 1554 from 109,041 households living in all 29 states (International Institute for Population Sciences 2007). The sample of NFHS-3 covers $99 \%$ of India's population. In addition to the nationally representative sample, NFHS-3 also collected socioeconomic and health information on slum and non-slum populations from eight major Indian cities.

In our study, we utilized a subset of the dataset $(N=17,239)$ to conduct our analyses. More specifically, we chose women who had a child between the ages of 2 and 5 $\left(M_{\mathrm{age}}=3.20, \mathrm{SD}_{\mathrm{age}}=.98\right)$ since stunting is unlikely to occur before the age of 2. Our sample of over 17,000 was sufficient to meet sample size requirements. On average, mothers were 20.17 years old $(\mathrm{SD}=3.98)$ at the time of their child's birth. About $42.5 \%$ of these families were from an urban area, while the remaining $57.5 \%$ were from a rural area.

\section{Measures}

Stunting At a theoretical level, we suggest that stunting among children is a function of religion, availability of toilets, mother's education, and source of water. The stunting scores are computed using the WHO age and gender-specific standards (WHO 1997). The reference population is derived from sample surveys the WHO conducted in 2006. The standardized stunting score, the dependent variable, is [ $\mathrm{Xi}-$ 
Xmedian)/SD] from the median of the reference population. Children with stunting scores less than -2 SD are labeled "stunted," and those with scores less than -3 SD are considered "severely stunted." Children with scores above -2 SD and well into the positive scores are not stunted. In our sample, children had an average stunting score of $-1.88(\mathrm{SD}=0.919)$ standard deviations below the median height. According to UNICEF, stunting is defined as a child measuring at least 2 standard deviations below the median height for age of the reference population; thus, this stunting variable was also dichotomized to create stunted (average stunting score of $<-2$ ) and non-stunted (average stunting score of $>-2$ ) groups. The stunting status of the child was measured using a dummy variable with 1 coded for "not-stunted" status and 0 for the rest. Consistent with the population, over $30 \%$ of the children in our sample was stunted by UNICEF definition $(n=5796)$.

Religion and Education Religion and maternal education were each assessed using one item on the survey. Due to the large population of Hindus in India, the variable of religion was dichotomized into Hindu (coded $1, n=12,512$ ) and nonHindu ( $n=4707)$ groups. Mothers had received an average of 5.47 years of education $(\mathrm{SD}=5.25)$. The variable was dichotomized, separating mothers who had completed a secondary education (more than 8 years; $n=6784$ ), coded 1 , from those who had not (less than 8 years; $n=10,455$ ), coded 0 .

Access to Sanitary Facilities and Clean Water Similarly, one item on the NFHS-3 survey assessed the respondents' access to sanitary bathroom facilities: flush, pit, and other types. Pit toilets are made up of a deep hole in the ground for collecting human excreta. Those who used a flush/pit type of toilet were coded 1. Over half of the sample $(n=9500)$ had access to a flushing or pit toilet, while the remainder $(n=6978)$ did not. The majority of respondents in this sample used a piped water source (coded $1, n=13,154$ ), while the others did not $(n=4085)$.

\section{Analytical Procedure}

In such a large-scale sample, missing data are expected. We found that $23.1 \%(n=3984)$ of our sample did not have data on the child's stunting. Missing values for stunting were imputed using the expectation-maximization method, a useful algorithm for computing maximum likelihood estimates from incomplete data (Dempster et al. 1977). All analyses involving the variable of stunting yielded similar results when using the imputed values, compared to analyses using the dataset with missing values and listwise deletion. Thus, the results described herein are for analyses conducted with data imputed for missing values. Due to the dichotomous nature of many of our variables, we utilized binary logistic regression for testing all the proposed hypotheses. The hypotheses stemming from the demographic and environmental sets examine the direct effects of the four determinants. The last hypothesis, however, investigates the moderating effect of "toilet-type" use on the influence of a mother's education on the likely stunting status of her child. This hypothesis requires a test of the interaction between the mother's education and the toilet type in use.

\section{Results}

In order to test our hypothesis that religion, education, access to a flushing/pit toilet, and piped water would predict stunting in children ages $2-5$, we employed a binomial logistic regression. Stunting was entered in as our dependent variable, and the dichotomous variables of religion (Hindu or non-Hindu), mother's education (completed secondary education or did not complete), access to sanitary facilities (flushing/pit toilet or other), and access to clean water (piped water or other) were entered in as our independent variables. In order to investigate the net effect of religion, we controlled for the caste effect using Deshpande's caste disparity index (CDI) (Deshpande 2001). We reversed the direction to align higher values of the index to higher levels of disparity. The disparity index was not available for all the Indian states. For states for which an index value was not available, we imputed the national value. Furthermore, for the two Indian states, Uttranchal and Jharkhand, which previously belonged to the state of Uttar Pradesh, we attributed the CDI for Uttar Pradesh.

Our results presented in Table 1 indicate that the odds of a Hindu child being not stunted are $6 \%$ more than the odds of being "not stunted" for a non-Hindu child. This does not support our hypothesis that Hindu children would have higher rates of stunting compared to their non-Hindu counterparts. However, the odds ratio is significant at the .1 level. We also found that the odds of children whose mothers have a secondary education are more than twice as likely to not be stunted compared to children whose mothers have not completed a secondary education, which supports our initial hypothesis.

Table 1 Demographic and environmental factors predicting stunting in children ages $2-5$

\begin{tabular}{llllll}
\hline Variables & $B$ & SE & Wald & Sig. & Exp $(B)$ \\
\hline RELIGION1 & .063 & .039 & 2.608 & .106 & 1.065 \\
MOTHEREDU & .920 & .038 & 575.397 & .000 & 2.508 \\
TOILET & 1.165 & .037 & $1.011 \mathrm{E} 3$ & .000 & 3.205 \\
H2OSOURCE & -.204 & .040 & 26.054 & .000 & .816 \\
Constant & -.508 & .049 & 105.815 & .000 & .602 \\
\hline
\end{tabular}

Variables are coded according to the following: religion $(1=$ Hindu, $0=$ non-Hindu), education $(1=$ completed secondary school, $0=$ did not complete), toilets $(1=$ access to flushing/pit, $0=$ other $)$, water $(1=$ access to pipe, $0=$ other $)$, stunting $(1=$ not stunted, $0=$ stunted $)$ 
We assess the adequacy of our logistic model with Hosmer and Lemeshow $\chi^{2}(7, N=12,672)=27.2, p=.000$, and Cox and Snell $R^{2}=.146$, Nagelkerke $R^{2}=.197$. The values of the model fit test suggest a lack of good fit with the observed data on stunting status. These results suggest that more theoretically relevant determinants are to be added to this model. Similarly, the odds of children with access to a flushing or pit toilet being "not stunted" are 3.5 times the odds of being "not stunted" for the rest of the children, which also supports our hypothesis. Children with access to piped water are $20 \%$ less likely to not be stunted than children who do not have access to a flush or pit toilet, which does not support our hypothesis that more sanitary water sources (piped) would predict less stunting among children between the ages of 2 and 5 .

In order to test our second hypothesis that access to flush and pit toilets and a mother having a secondary education predict lower rates of childhood stunting among children between the ages of 2 to 5 , we conducted a 2 (toilet type: flushing or non-flushing) by 2 (maternal education: secondary or less than secondary) factorial analysis of variance (ANOVA). See Table 2. The assumptions of sample representativeness, normality of distribution, and sample sizes were tested and met prior to analysis. The test for homoscedasticity, Levene's test, proved to be significant, $F(3,12,767)=16.14$, $p<.001$, and the observed power for the analysis was adequate $((1-\beta)>.82)$. Results from the ANOVA indicated a significant main effect of maternal education, $F(1$, $12,767)=200.16, p<.001, \eta_{p}{ }^{2}=.02$. Post hoc pairwise comparisons with a Bonferroni adjustment illustrated that children of mothers who have completed a secondary education are significantly less stunted than those whose mothers did not finish secondary school (see Table 2). The main effect of toilet type was also significant, $F(1,12,767)=140.75, p<.001, \eta_{p}^{2}=.01$; children with access to more sanitary toilet facilities (flush and pit) were less

Table 2 Levels of stunting in children ages 2 to 5 by toilet type and maternal education

\begin{tabular}{lll}
\hline Category & $M$ & SE \\
\hline Access to flush/pit toilet & -1.75 & .01 \\
Access to other type of toilet & -2.01 & .02 \\
Non-educated mother (did not complete secondary school) & -2.03 & .01 \\
Educated mother (completed secondary school) & -1.72 & .02 \\
Flush/pit toilet and non-educated mother & -1.93 & .02 \\
Flush/pit toilet and educated mother & -1.56 & .02 \\
Other toilet and non-educated mother & -2.13 & .02 \\
Other toilet and educated mother & -1.88 & .03
\end{tabular}

Levels of stunting are $z$-scores (standardized values). Lower (more negative) scores indicate more stunting (i.e., lower weight/height)

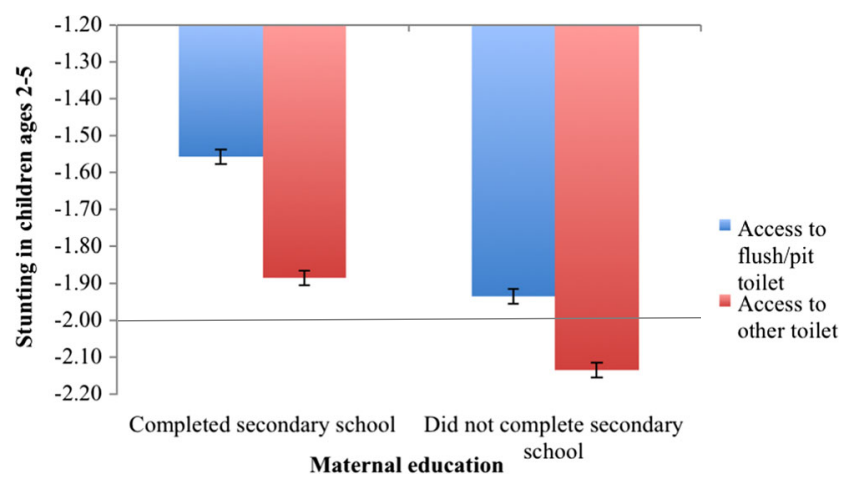

Fig. 1 Comparison of levels of stunting in children ages 2-5 for maternal education and access to toilet facilities. Standardized scores are shown for stunting in children. The line at -2.00 ( 2 standard deviations below the mean) represents the cutoff for "stunted growth" according to UN guidelines

stunted than children without such access (Table 2). Furthermore, the interaction between toilet type and maternal education was also significant, $F(1,12,767)=8.35, p=.004$, $\eta_{p}{ }^{2}=.001$. Children with access to flushing or pit toilets whose mothers had completed a secondary education were the least stunted, while children without access to sanitary facilities whose mothers were not educated had higher rates of stunting. See Fig. 1.

The significant Hosmer-Lemeshow statistics as well as the lack of support for the theoretical expectation of significant difference in stunting status between Hindus and non-Hindus may be due to loss of information stemming from the categorization of stunting status into a dummy variable. This supposition calls for the application of a linear regression approach assuming that the dependent variable "stunting" is normally distributed. Table 3 presents the results of regression of "stunting" on all the selected independent variables including the interaction variable capturing the moderating effect of toilet type on the relationship between a mother's education level and the stunting status of her children. The effect of the variable "religion" is significant but has a sign contrary to the expected. We repeated the analysis controlling for the effect of caste disparity. The effect of CDI on stunting was negative, higher levels of disparity was significantly associated with higher levels of stunting. However, even after controlling the effect of caste on stunting, the net effect of religion remained significant at the $10 \%$ level and in the same direction.

The mean difference between Hindus and non-Hindus is significant and is positive suggesting that Hindu children have higher scores on the stunting status variable than non-Hindus. As higher stunting scores suggest movement toward non-stunting status, our results indicate that Hindu children are less likely to be stunted than non-Hindu children. The mother's education as expected has a positive effect on stunting scores. 
Table 3 Regression of stunting scores (standardized measure) on selected determinants

\begin{tabular}{|c|c|c|c|c|c|}
\hline \multirow[t]{2}{*}{ Model } & \multicolumn{2}{|c|}{ Unstandardized coefficients } & \multirow{2}{*}{$\begin{array}{l}\text { Standardized } \\
\text { coefficients } \\
\text { Beta }\end{array}$} & \multirow[t]{2}{*}{$t$} & \multirow[t]{2}{*}{ Sig. } \\
\hline & $B$ & Std. error & & & \\
\hline (Constant) & -2.103 & .021 & & -102.398 & .000 \\
\hline Religion1 & .033 & .016 & .016 & 2.087 & .037 \\
\hline MotherEdu & .267 & .030 & .142 & 8.988 & .000 \\
\hline Toilet & .196 & .018 & .105 & 10.977 & .000 \\
\hline H2osource & -.078 & .016 & -.036 & -4.904 & .000 \\
\hline Interaction & .119 & .035 & .061 & 3.447 & .001 \\
\hline
\end{tabular}

Dependent variable: hw3_1a (standardized measure of stunting). Variables are coded according to the following: religion1 $(1=$ Hindu, $0=$ non-Hindu $)$, education $(1=$ completed secondary school, $0=$ did not complete), toilets $(1=$ access to flushing/pit, $0=$ other $)$, water $(1=$ access to pipe, $0=$ other $)$, and stunting (standardized measure)
Those who have a "pit/flush" toilet type also have significantly better stunting scores than the rest as expected. The next hypothesis that households with "pipe/tube water" are more likely to have higher stunting scores than those who do not have "pipe/tube" water is not supported. The variable is significant and the relationship is negative rather than positive as hypothesized. The last hypothesis related to the interaction between the "toilet type" used and the "mother's education" level was supported. Mothers with a secondary level of education with the "pit/flush" toilet type are significantly more likely than the rest to have higher stunting scores. Thus, children of these mothers are less likely to be stunted than the children of the rest of the mothers. In general, the results from the multiple regression model of stunting are similar to the results from the logistic regression model while enabling us to account for the full variation in the stunting scores.

Lastly, we found that children with mothers that have a primary education (not completed secondary school) were more likely to have a toilet. See Table 4. Also, Hindu children were less likely to have a toilet than their non-Hindu counterparts. Our results suggest that Hindus in general are much more likely to suffer from poor sanitary conditions outside their home. However, as stated above, Hindus compared to non-Hindus were less stunted. This sheds light on factors that are contributing to stunting in India, besides unhygienic toilet facilities and water supply.

Table 4 Maternal education and religion predicting access to toilet facilities

\begin{tabular}{lrllllll}
\hline Predictor & $b$ & SE & Wald & $p$ & OR & LLCI & ULCI \\
\hline Religion & 2.14 & .04 & 2741.63 & $<.001$ & 8.49 & 7.83 & 9.20 \\
Mother's education & -1.19 & .04 & 811.20 & $<.001$ & .30 & .28 & .33 \\
\hline
\end{tabular}

\section{Discussion}

In recent years, it has become clear among policymakers and global health experts that nutritional interventions are only part of the solution to stunted growth among children throughout the world. In places like India, where almost half of the children under 5 years of age are stunted, continued research in understanding the socioeconomic variables on stunting is critical. Therefore, the focus of this paper was to gain a better understanding of how sanitary practices, drinking water, mother's education, and religion impact childhood stunting rates and toilet access/use.

Our study finds that stunting is not only about nutrition; it is also about cultural practices and water supply. Studies in Bangladesh, Sudan, and Mexico showed that there was a strong association between impaired linear growth and ongoing diarrhea (Schmidt 2014). However, researchers in Karachi, Pakistan, found that hand washing by children and their parents was highly protective against diarrhea but had no effect on stunting (Ngure et al. 2014). Therefore, this suggests that safe drinking water alone may not reduce childhood stunting but other socioeconomic factors such as educational interventions to women regardless of religion and sanitary toilet facilities are vital to the reduction of stunted children.

Since both water and religion have been controversial variables in the literature on stunting, our findings on these two variables are consistent with recent analysis that suggests that action must be taken across multiple areas to reduce stunting. In our second hypothesis, we also measured socioeconomic variables' impact on stunting; however, stunting was measured on a continuous level. As expected, the children of mothers with a secondary education had lower stunting rates than the children of mothers without a secondary education. Also, children with access to sanitary toilets had a lower rate of stunting than those without. Note also that children who had mothers with a secondary education and access to sanitary toilet facilities were the least stunted and children who had 
neither a mother that had a secondary education nor access to sanitary toilet facilities had the highest levels of stunting. Our finding is consistent with recent research that suggests the reduction of stunting requires direct interventions that focus on both poor sanitation and hygiene. However, further research should be done on the role that the mother's education plays in influencing household practices such as hand washing, food intake, and resource management.

Research on how socioeconomic status, education, nutrition, and religion interact would provide a better understanding on how to influence policy on both the national and local levels. This would also positively impact the internal and external funding necessary to tackle a systematic issue that is robbing the lives of innocent children and their families.

Our results provide insights into policy approaches for improving malnutrition in India. Current governmental policies and programs for eradicating malnutrition among children in India fall broadly under two categories. A set of programs in the first category provides both direct as well as ancillary support services. A recent evaluation of these programs provides only scant evidence of achievement of expected program outcomes (Singh et al. 2014). A second category of programs is anchored on a set of assumptions that indirectly link malnutrition with a number of environmental determinants associated with sanitary conditions. Both categories of programs appear to an extent to ignore the social and human capital mothers bring to bear on the health of their children. Our study found that mothers' characteristics such as education have a significant effect on reducing the level of stunting. The mother's capacity to make intrahousehold allocations for safeguarding children's adequate nutritional intake is crucial for the success of prevention programs against child malnutrition. When mothers are constrained in shifting intrahousehold resource allocations due to cultural or social constraints, malnutrition is more likely to occur.

Though we have emphasized the salient contributions of the mother's characteristics such as education on reducing levels of stunting among children, our study underscores the importance of the indirect effects of environmental factors on stunting levels. Demand for various toilet types is dependent upon not only the source of water supply but also the cultural attributions of distance from waste matter including excreta. Both factors influence the likelihood of use of flush toilets. These variables influencing toilet type have indirect effects on nutritional levels. Infrastructural development ensuring adequate water supply to the household may be pursued hand in hand with women's empowerment programs to decrease the likelihood of stunting among Indian children.

Religious values provide a basis for consensus on the need to preserve children's health. We found differences in stunting across religions. Both values and the structures through which values are disseminated may make a difference. Therefore, in order to promote children's health, social workers have to work toward seeking and reaching common grounds with regard to the need to ensure children's health (Hall and Elliman 2006; Banks 2012). We also found that structural barriers imposed by intercaste distances negatively affect stunting. Though the role of the caste on a multitude of outcomes such as educational and occupational mobility (Jalan and Murgai 2007) has been studied, its effect on children's health has not received adequate attention (Kabeer 2006). Advocating equal representation of caste groups in all political and civil organizations will empower oppressed groups to reduce power differentials and create a social environment supportive of children's health. A mother's education increases her capabilities to improve children's health. Mothers use their improved awareness and knowledge gained through education in improving children's health. Finally, availability of flush toilets and its significance for prevention of stunting underscore the importance of controlling physical environments for realizing health as a right for all children.

In general, our results suggest a three-pronged approach toward achieving children's health as a right of every child. First, reduction of differences between the social and religious values with respect to children's health will have to be realized through consensus building (George et al. 2000). Second, the economic basis for forging consensus with respect to the desirability and importance of children's health should not be overlooked. That is, opportunities for seeking outcomes as a matter of individual rights call for the organization of production and distribution of resources for sustenance organization of the community. Economic structural barriers that limit the achievement of right have to be addressed within the context of livelihood and sustenance organization of the community. Finally, human rights outcomes involve improving capabilities (Sen 2005) as in the case of improvement of the mother's education with positive effects on children's health.

Thus, a combination of economic- and capability-oriented approaches along with value changes through community organization and advocacy is necessary for stunting prevention. One important contribution of this study involves the development of an empirical approach toward developing interventions based on a human rights approach. More specifically, this approach may be replicated for developing social work interventions for any number of social problems, which may be cast as a human rights issue.

The Universal Declaration of Human Rights, and the Convention of the Rights of the Children, and several other international organizations have recognized that it is a right to not be malnourished; however, very few nations, including the USA, have legislatively addressed the issue (Donoho 2006). Historically, human rights included such demands as personal liberty and political freedom, although controversial "secondary rights," such as economic and social rights, are now considered to be within the broader class of human rights (Wronka 2007). Therefore, codifying and legitimizing institutional governmental actions that address economic and social 
rights are necessary for political and societal change in a country that has largely ignored an epidemic that robs its most vulnerable members of society - children. Reducing childhood stunting by protecting the basic rights of children and respectfully challenging age-old cultural and religious practices by providing sound educational opportunities about the dangers of open defecation and unhygienic sanitation practices will potentially save India from losing its most valuable resource - growing minds and hearts.

The limitations of this study need to be considered. It is important to note that although the mother's educational level was a salient finding, the variable was dichotomized for simplicity. More information on the levels of education would have provided a clearer understanding of how education impacts activities of daily living such as toilet use. Furthermore, only four socioeconomic variables were selected in this analysis. There are several other sociocultural and socioeconomic variables that can potentially impact childhood stunting. Although recent literature suggests that childhood stunting is not caused by dietary insufficiencies and inadequacies alone, economic, social, and environmental practices and structures play a critical role and examining the interaction of nutritional variables with socioeconomic variables may have provided a better understanding on how nutrition-specific interventions coupled with a broader framework could be implemented. Despite these limitations, accessing key socioeconomic variables with childhood stunting using a large representative local survey data set is critical in providing governments, the primary agents for the realization of human rights, an opportunity to develop targeted interventions that reduce stunting rates among Indian children.

It is suggested that future studies on stunting in India focus on the unique behaviors of women that have a primary education or higher and the interaction with sanitary practices on childhood stunting. Further research in this area may be helpful in developing education programs that can be integrated with meaningful sanitation programs for women across cultural, religious, and socioeconomic groups. Although, in our study, a positive relationship between women's higher education and the reduced level of stunting was found, it is important to note that women's access to education (primary and secondary) alone does not translate into her ability to have decision-making power and control of household resources. Thus, while educational access is critical in ensuring women are empowered, it alone is not sufficient. Without women's ability to recognize and utilize the strength of this resource, it cannot bring about empowerment. Therefore, women's empowerment is a complex phenomenon that involves multiple cultural, educational, and economic shifts. Further research in this area may be helpful in developing multipronged interventions in reducing the incidence of stunting among children.

\section{References}

Aguayo, V. M., Nair, R., Badgaiyan, N., \& Krishna, V. (2016). Determinants of stunting and poor linear growth in children under 2 years of age in India: an in-depth analysis of Maharashtra's comprehensive nutrition survey. Maternal \& Child Nutrition, 12(S1), $121-140$.

Banda, K., Sarkar, R., Gopal, S., Govindarajan, J., Harijan, B. B., Jeyakumar, M. B., \& Thomas, V. A. (2007). Water handling, sanitation and defecation practices in rural southern India: a knowledge, attitudes and practices study. Transactions of the Royal Society of Tropical Medicine and Hygiene, 101(11), 1124-1130.

Banks, S. (2012). Ethics and Values in Social Work. Palgrave Macmillan.

Bhalotra, S., Valente, C., \& van Soest, A. (2010). The puzzle of Muslim advantage in child survival in India. Journal of Health Economics, 29(2), 191-204.

Bose, K., Biswas, S., Bisai, S., Ganguli, S., Khatun, A., Mukhopadhyay, A., \& Bhadra, M. (2007). Stunting, underweight and wasting among Integrated Child Development Services (ICDS) scheme children aged 3-5 years of Chapra, Nadia District, West Bengal, India. Maternal \& Child Nutrition, 3(3), 216-221.

Brainerd, E., \& Menon, N. (2015). Religion and health in early childhood: evidence from South Asia. Population and Development Review, 41(3), 439-463.

Chaudhury, P. (2004). The 'creamy layer': political economy of reservations. Economic and Political Weekly, 1989-1991.

Dempster, A.P., Laird, N.M., \& Rubin, D.B. (1977). Maximum likelihood from incomplete data via the EM algorithm. Journal of the Royal Statistical Society. Series B (Methodological), 1-38.

Deshpande, A. (2001). Caste at birth? Redefining disparity in India. Review of Development Economics, 5(1), 130-144.

Donoho, D. (2006). Human rights enforcement in the twenty-first century. Ga. J. Int'l \& Comp. L., 35, 1.

George, L. K., Larson, D. B., Koenig, H. G., \& McCullough, M. E. (2000). Spirituality and health: what we know, what we need to know. Journal of Social and Clinical Psychology, 19(1), 102.

Geruso, M., \& Spears, D. (2014). Sanitation and health externalities: resolving the Muslim mortality paradox. University of Texas at Austin Working Paper.

Gulati, J. K. (2010). Child malnutrition: trends and issues. Anthropologist, 12(2), 131-140.

Haddad, L., Nisbett, N., Barnett, I., \& Valli, E. (2014). Maharashtra's child stunting declines: what is driving them? Findings of a Multidisciplinary Analysis.

Hall, D.M., \& Elliman, D. (2006). Health for all children: revised fourth edition. Oxford University Press.

International Institute for Population Sciences (2007). India National Family Health Survey (NFHS-3), 2005-06 (Vol 1). International Institute for Population Sciences.

Jalan, J., \& Murgai, R. (2007). Intergenerational mobility in education in India. Delhi: the World Bank. Processed.

Kabeer, N. (2006). Poverty, social exclusion and the MDGs: the challenge of 'durable inequalities' in the Asian context. IDS Bulletin, 37(3), 64-78.

Kanjilal, B., Mazumdar, P. G., Mukherjee, M., \& Rahman, M. H. (2010). Nutritional status of children in India: household socio-economic condition as the contextual determinant. International Journal for Equity in Health, 9(1), 19.

Maruthi, I., \& Busenna, P. (2015). Drinking water, sanitation and hygiene practices of Dalits in Karnataka. Journal of Governance \& Public Policy, 5(2), 82.

Ngure, F. M., Reid, B. M., Humphrey, J. H., Mbuya, M. N., Pelto, G., \& Stoltzfus, R. J. (2014). Water, sanitation, and hygiene (WASH), environmental enteropathy, nutrition, and early child development: making the links. Annals of the New York Academy of Sciences, 1308(1), 118-128. 
Panagariya, A. (2013). Does India really suffer from worse child malnutrition than Sub-Saharan Africa? Economic and Political Weekly, 48(18), 98-111.

Pillai, V.K., \& Parekh, R. (2015). Sanitation and social change. Yojana: Journal of the Ministry of Planning (India), 7-14.

Schmidt, C. W. (2014). Beyond malnutrition: the role of sanitation in stunted growth. Environmental Health Perspectives, 122(11), A298.

Sen, A. (2005). Human rights and capabilities. Journal of Human Development, 6(2), 151-166.

Shah, D. J., \& Bali, S. (2015). Study of nutritional status and identification of associated risk factors in children below five years of age in an urban slum of Bhopal, Madhya Pradesh. Indian Journal of Community Health, 27(4), 504-508.

Singer, M., \& Erickson, P. I. (2013). Global health: an anthropological perspective. Waveland Press.

Singh, A., Park, A., \& Dercon, S. (2014). School meals as a safety net: an evaluation of the Midday Meal Scheme in India. Economic Development and Cultural Change, 62(2), 275-306.
Som, S., Pal, M., \& Bharati, P. (2007). Role of individual and household level factors on stunting: a comparative study in three Indian states. Annals of Human Biology, 34(6), 632-646.

Spears, D., Ghosh, A., \& Cumming, O. (2013). Open defecation and childhood stunting in India: an ecological analysis of new data from 112 districts. PLoS ONE, 8(9), e73784.

Stillwaggon, E. (1998). Stunted lives, stagnant economies: poverty, disease, and underdevelopment. Rutgers University Press.

Thorat, S., \& Newman, K.S. (2007). Caste and economic discrimination: causes, consequences and remedies. Economic and Political Weekly, $4121-4124$.

Victora, C. G., Adair, L., Fall, C., Hallal, P. C., Martorell, R., Richter, L., Sachdev, H. S., \& Maternal \& Child Undernutrition Study Group. (2008). Maternal and child undernutrition: consequences for adult health and human capital. The Lancet, 371(9609), 340-357.

WHO. (1997). WHO global database on child growth and malnutrition. Geneva: WHO.

Wronka, J. (2007). Human rights and social justice: social action and service for the helping and health professions. Sage. 